\title{
Enhanced weighted method for test case prioritization in regression testing using unique priority value
}

\begin{abstract}
Regression testing is an integral and expensive part in software testing. To reduce its effort, test case prioritization approaches were proposed. The problem with most of the existing approaches is the random ranking of test cases with equal weight. In this paper, an enhanced weighted method to prioritize the full test suite without using random ranking is presented. In addition, a controlled experiment was executed to evaluate the effectiveness of the proposed method. The results show an improved performance in terms of prioritizing test cases and recording higher APFD values over the original weighted method. In future, a larger experiment would be executed to generalize the results.
\end{abstract}

Keyword: Regression testing; Test case prioritization; Weighted method 